\title{
Moments of multiplicity distributions using Tsallis statistics in leptonic and hadronic collisions
}

\author{
S. Sharma* and M. Kaur \\ Department of Physics, Panjab University, Chandigarh 160 014, India
}

(Received 28 March 2019; published 16 May 2019)

\begin{abstract}
Moments play a crucial role in investigating the characteristics of charged particle multiplicities in highenergy interactions. The success of any model which describes the multiplicity data can be understood well by studying the normalized and factorial moments of that distribution. The Tsallis model is one of the most successful models which describes the multiplicity spectra and transverse momentum $\left(p_{T}\right)$ spectra very precisely in high-energy interactions. In our previous work, we used the Tsallis $q$ statistics to describe the multiplicity distributions in leptonic and hadronic collisions at various energies ranging from $14 \mathrm{GeV}$ to $7 \mathrm{TeV}$. In the present study, we have extended our analysis for calculating the moments using the Tsallis model for $e^{+} e^{-}$interactions at $\sqrt{s}=91$ to $206 \mathrm{GeV}$ from the large electron-positron collider data and for $p p$ interactions at $\sqrt{s}=0.9$ to $7 \mathrm{TeV}$ in various pseudorapidity intervals from the CMS data at the LHC. By using the Tsallis model, we have also calculated the average charged multiplicity and its dependence on energy. It is found that the moments and the mean multiplicities predicted by the Tsallis model are in good agreement with the experimental values. We have also predicted the mean multiplicity at $\sqrt{s}=500 \mathrm{GeV}$ for $e^{+} e^{-}$collisions and at $\sqrt{s}=14 \mathrm{TeV}$ for $p p$ collisions in the extreme pseudorapidity interval, $|\eta|<2.4$.
\end{abstract}

DOI: 10.1103/PhysRevD.99.096016

\section{INTRODUCTION}

In high-energy collisions, particles are made to collide with relativistic momenta much greater than their rest masses, resulting in the production of a large number of particles in the final state [1] from a variety of processes. These collisions can be hadronic, leptonic, or heavy-ion interactions, summarized in the form of reaction, for leptonic collision as $l-l \rightarrow X$ [2], where $l$ is the lepton or for hadronic collisions as $h-h \rightarrow X$ [3], where $h$ is the hadron or for hadron-nucleus collision as $h-A \rightarrow X$ [4], with $A$ being the nucleus. $X$ in the final state of these reactions represents any number of particles, produced due to the gluon-gluon, quark-quark, and quark-gluon interactions between the constituent quarks and gluons of the colliding particles. The produced particles can be the baryons (qqq state), mesons ( $\bar{q} q$ state), or leptons. The simplest but the most significant observation to describe the mechanism of particle production is the observation of charged particle multiplicity $[5,6]$ and the distribution of the number of particles produced, known as multiplicity distribution [7]. Multiplicity distribution (MD) also carries

\footnotetext{
*sandeep.sharma.hep@gmail.com
}

Published by the American Physical Society under the terms of the Creative Commons Attribution 4.0 International license. Further distribution of this work must maintain attribution to the author(s) and the published article's title, journal citation, and DOI. Funded by SCOAP ${ }^{3}$. important information about the correlations of particles produced, thus providing a very fine way to investigate the dynamics of the quark-quark, gluon-gluon, and quarkgluon interactions.

Collision or interaction of two particles is generally described in terms of a cross section, which is calculated by measuring the number of particles produced. The cross section essentially gives the measure of the probability of production of a particular number of particles. The multiplicity distribution is defined in terms of probability by the formula

$$
P_{N}=\frac{\sigma_{N}}{\sigma_{\text {total }}}=\frac{N_{\mathrm{ch}}}{N_{\text {total }}}
$$

where $\sigma_{N}$ is the cross section for the production of $N$ number of particles and $\sigma_{\text {total }}$ represents the total cross section of interaction at center-of-mass energy $\sqrt{s}$. Experimentally, this probability, $P_{N}$, is obtained from the number of charged particles produced at specific multiplicity, $N_{\mathrm{ch}}$, and the total number of particles produced during the collisions, $N_{\text {total }}$. The MD obeys conventional Poisson distribution [8] if there is no correlation between the particles produced; i.e., particles produced are exclusive and independent of each other [9]. The presence of any kind of correlation among the particles leads to the deviation from Poissonian form. Higher-order moments and their cumulants are the precise tools to study the correlation between the particles produced in these interactions $[10,11]$. 
In the last few years, the Tsallis model [12] has been used successfully in describing the MDs in hadronic and leptonic collisions for different collision energies. Recently, we have analyzed the $e^{+} e^{-}, p p$, and $\bar{p} p$ collisions at different energies by using the Tsallis model [13-16]. In the present study, we use the Tsallis approach to measure correlations between the particles produced in both leptonic and hadronic interactions at energies ranging from a few giga-electron-volts up to the LHC energies. Additionally, the dependence of the average multiplicity on the center-of-mass energy is also studied. Results from the Tsallis model are compared with experimental values. In Sec. II, we give a brief description of moments and the formulation to calculate the higher-order moments. Section III gives the details of the data used and results obtained from the Tsallis model and its comparison with the experimental values. The method of calculation of uncertainty on the normalized and factorial moments is discussed in Sec. IV. The discussion and conclusion are presented in Sec. V.

\section{MOMENTS}

Multiplicity distributions at low energies of approximately $10 \mathrm{GeV}$ for leptonic and hadronic collisions such as $e^{+} e^{-}$or $p p$ could be described very well using Poisson distribution $[8,17]$. In such cases, dispersion, defined by $D=\sqrt{\left\langle N^{2}\right\rangle-\langle N\rangle^{2}}$, is related with the average multiplicity $\langle N\rangle$. The multiplicity distributions exhibited a broader width at higher energies, showing the significant deviation from the Poissonian form. The correlation in the particles produced during the collisions was found to be responsible for the deviations. The shape of multiplicity distribution can be described well using the assumption that energy dependence of multiplicity distribution at higher energies could be formulated using the average multiplicity. To explain the energy dependence of multiplicity, Koba et al. [18] in 1972 proposed the scaling relation for multiplicity distributions known as KNO scaling. It is the theory of universal scaling for multiplicity distributions in the asymptotic limit of energy. The energy dependence of the dispersion defined by relation $D \propto\langle N\rangle$ implied the compliance of KNO scaling. But a few years later, violation of KNO scaling was observed by the UA5 Collaboration while analyzing the multiplicity data at $\sqrt{s}=540 \mathrm{GeV}$ [19] obtained from $\bar{p} p$ collisions. Later on, it was shown by the collaboration that $\mathrm{KNO}$ scaling was violated even at $\sqrt{s}=200 \mathrm{GeV}$ [20]. Higher-order moments and its cumulants are the precise tools to study the correlations between the particles produced in collisions [21,22]. The departure from independent and uncorrelated production of particles can be measured well using the factorial moments, $F_{m}$ [23]. Not only the correlation between the particles but also the violation or holding of KNO scaling at higher energies can also be studied and understood correctly by using the normalized moments of order $m, C_{m}$ [24]. These moments are defined as

$$
\begin{gathered}
C_{m}=\frac{\left\langle N^{m}\right\rangle}{\langle N\rangle^{m}} \\
F_{m}=\frac{\langle(N(N-1) \ldots(N-m+1))\rangle}{\langle N\rangle^{m}} .
\end{gathered}
$$

The factorial moments and their cumulants, $K_{m}$, are near to precise in defining the tail part of distribution where events with a multitude of particles give a meaningful contribution. The factorial moments and cumulants are related to each other by the relation

$$
F_{m}=\sum_{i=0}^{m-1} C_{m-1}^{i} K_{m-i} F_{i} .
$$

Factorial moments exhibit the features of any kind of correlation present between the particles and cumulants of order $m$ illustrate absolute $m$-particle correlation which cannot be brought down to the lower-order correlation. In other words, if all $m$ particles are related to each other in $m$ th order of cumulants, then it cannot be divided into disconnected groups, i.e., the $m$ particle cluster cannot be split in to smaller clusters. These moments and their dependence on energy $\sqrt{s}$ help in improving, redefining, and rejecting various Monte Carlo or statistical models, which can be used in describing the production of particles at high energies.

\section{A. Tsallis distribution}

Tsallis statistics [12] uses the concept of the nonextensive nature of entropy, which is the modification of the usual Boltzman-Gibbs [25] and is given by

$$
S=\frac{1-\sum_{a} P_{a}^{q}}{q-1}
$$

where $P_{a}$ is the probability associated with microstate $a$ and the sum of the probabilities over all microstates is normalized: $\sum_{a} P_{a}=1$. For the entropic index $q$ with value $q>1,1-q$ measures the departure of entropy from its extensive behavior.

The probability distribution function in the case of Tsallis $q$ statistics is defined using the partition function $\mathrm{Z}$ as

$$
P_{N}=\frac{Z_{q}^{N}}{Z}
$$

where $\mathrm{Z}$ represents the total partition function and $Z_{q}^{N}$ represents partition function at a particular multiplicity. For $\mathrm{N}$ particles, the partition function can be written as 


$$
Z(\beta, \mu, V)=\sum\left(\frac{1}{N !}\right)\left(n V-n v_{0} N\right)^{N}
$$

$n$ represents the gas density, $\mathrm{V}$ is the volume of the system, and $v_{0}$ is the excluded volume. The generating function of the distribution plays an important role in providing the physical information of the multiplicity distribution. The generating function for multiplicity distribution is related to the probability as

$$
G(t)=\sum_{N=0}^{\infty} P_{N} t^{N}
$$

and can be obtained by using the expression of probability distribution function as given by

$$
\begin{aligned}
G(t) \approx & \exp (t-1) V n\left[1+(q-1) \lambda(V n \lambda-1)-2 v_{0} n\right] \\
& +(t-1)^{2}(V n)^{2}\left[(q-1) \frac{\lambda^{2}}{2}-\frac{v_{0}}{V}\right] .
\end{aligned}
$$

The Tsallis probability generating function has the same form as that of the negative binomial distribution $\left(G_{N B D}=\left[1-\frac{\langle N\rangle}{k}(t-1)\right]^{-k}=\exp [\langle N\rangle(t-1)]\right)$ with the average of number of particles $\bar{N}$ for the Tsallis probability as

$$
\bar{N}=V n\left[1+(q-1) \lambda(V n \lambda-1)-2 v_{0} n\right],
$$

where $\lambda$ is related to the temperature through parameter $\lambda$ as

$$
\lambda(\beta, \mu)=-\frac{\beta}{n} \frac{\partial n}{\partial \beta} .
$$

More details about the calculations can be found in Refs. [16,26].

\section{B. Moments of the Tsallis distribution}

The normalized moments of order $m$ of the Tsallis distribution can be calculated through the average number of particles using $C_{m}=\frac{\left\langle N^{m}\right\rangle}{\langle N\rangle^{m}}$, where average multiplicity $\bar{N}=\langle N\rangle$. The factorial moments are defined as

$$
\begin{gathered}
F_{m}=\frac{\langle(N(N-1) \ldots(N-m+1))\rangle}{\langle N\rangle^{m}} \\
F_{m}=\left(\frac{1}{\langle N\rangle^{m}}\right) \frac{d^{m} G(t)}{d t^{m}},
\end{gathered}
$$

where $G(t)$ is the generating function of the Tsallis distribution defined by Eq. (9). The factorial moments are related to the normalized moments and can be written in the terms of $C_{m}$. The first five factorial moments are

$$
\begin{aligned}
& F_{2}=C_{2}-\frac{C_{1}}{\langle N\rangle} \\
& F_{3}=C_{3}-3 \frac{C_{2}}{\langle N\rangle}+2 \frac{C_{1}}{\langle N\rangle^{2}} \\
& F_{4}=C_{4}-6 \frac{C_{3}}{\langle N\rangle}+11 \frac{C_{2}}{\langle N\rangle^{2}}-6 \frac{C_{1}}{\langle N\rangle^{3}} \\
& F_{5}=C_{5}-10 \frac{C_{4}}{\langle N\rangle}+35 \frac{C_{3}}{\langle N\rangle^{2}}-50 \frac{C_{2}}{\langle N\rangle^{3}}+24 \frac{C_{1}}{\langle N\rangle^{4}} .
\end{aligned}
$$

\section{RESULTS}

Experimental data of proton-proton collisions from the CMS experiment at the Large Hadron Collider and the data of $e^{+} e^{-}$annihilation at different collision energies from the OPAL and L3 experiments are analyzed. The $p p$ data are analyzed at $\sqrt{s}=0.9,2.34,7 \mathrm{TeV}$ in the restricted pseudorapidity windows of $|\eta|<0.5,1.0,1.5,2.0,2.4$ [27]. The leptonic data from the L3 and OPAL experiments at $\sqrt{s}=91$ to $206 \mathrm{GeV}$ [28-32] in the full phase space are analyzed. Various analyses on the multiplicity distributions using these data have been done by us previously, and results can be found in Refs. [13-16]. In the following sections, results of the moments and average multiplicities obtained using the Tsallis statistics at different energies are discussed.

\section{A. Average multiplicities}

The energy dependence of mean charged multiplicity $\langle N\rangle$ is expected to reflect the underlying particle production process. A number of phenomenological models have been proposed to describe the behavior of mean charged multiplicity with energy. One of the most widely accepted relations that describes the multiplicity as a function of energy $\sqrt{s}$ is [33]

$$
\langle N\rangle=a+b \ln (\sqrt{s})+\operatorname{cln}^{2}(\sqrt{s}) .
$$

We calculate the values of average charged multiplicity from the Tsallis model and compare with the experimental values for $e^{+} e^{-}$collision data and for $p p$ collision data. The $\langle N\rangle$ calculated from the Tsallis model are given in Tables I and II. The values are found to be in good agreement with the experimental values, taking the errors into account.

Figure 1 shows the comparison of $\langle N\rangle$ values from the data and the Tsallis model for $e^{+} e^{-}$collisions at different center-of-mass energies, with $\sqrt{s}$ expressed in gigaelectron-volts. The data are also fitted to Eq. (15) as given below.

For data,

$$
\langle N\rangle=176.74-70.52(\ln \sqrt{s})+7.99(\ln \sqrt{s})^{2} .
$$


TABLE I. Average multiplicity $\langle N\rangle$ at $\sqrt{s}=91$ to $206 \mathrm{GeV}$ for $e^{+} e^{-}$interactions. The values obtained from the Tsallis model are compared with the OPAL and the L3 experimental values.

\begin{tabular}{lccc}
\hline \hline \multirow{2}{*}{ Experiment } & & \multicolumn{2}{c}{ Average charged multiplicity $\langle N\rangle$} \\
\cline { 2 - 4 } & Energy & Experimental & Tsallis model \\
\hline OPAL & 91 & $21.40 \pm 0.43$ & $21.07 \pm 0.21$ \\
& 133 & $23.40 \pm 0.65$ & $23.17 \pm 0.29$ \\
& 161 & $24.46 \pm 0.63$ & $24.01 \pm 0.47$ \\
& 172 & $25.77 \pm 1.05$ & $24.98 \pm 0.53$ \\
& 183 & $26.85 \pm 0.58$ & $26.17 \pm 0.39$ \\
& 189 & $26.95 \pm 0.53$ & $26.33 \pm 0.66$ \\
L3 & & & \\
& 130.1 & $23.28 \pm 0.26$ & $23.21 \pm 0.35$ \\
& 136.1 & $24.13 \pm 0.29$ & $23.53 \pm 0.17$ \\
& 172.3 & $27.00 \pm 0.58$ & $26.93 \pm 0.25$ \\
& 182.8 & $26.84 \pm 0.34$ & $26.77 \pm 0.19$ \\
& 188.6 & $26.84 \pm 0.32$ & $26.51 \pm 0.08$ \\
& 194.4 & $27.14 \pm 0.42$ & $26.87 \pm 0.49$ \\
& 200.2 & $27.73 \pm 0.47$ & $27.09 \pm 0.31$ \\
& 206.2 & $28.09 \pm 0.33$ & $27.38 \pm 0.20$ \\
\hline \hline
\end{tabular}

For the Tsallis model,

$$
\langle N\rangle=134.85-53.11(\ln \sqrt{s})+6.183(\ln \sqrt{s})^{2} .
$$

In the case of $p p$ collision data, the extreme pseudorapidity regions, $|\eta|<0.5$ and 2.4 , are chosen because of the availability of $\langle N\rangle$ values for these pseudorapidities only. Figure 2 shows the comparison of $\langle N\rangle$ values from the data and the model at $|\eta|<2.4$. In this case, the empirical relation describing the dependence of $\langle N\rangle$ on the center-ofmass energy, with $\sqrt{s}$ in tera-electron-volts takes the form as follows.

For data,

$$
\langle N\rangle=18.77+4.39(\ln \sqrt{s})+0.845(\ln \sqrt{s})^{2} .
$$

For the Tsallis model,

$$
\langle N\rangle=19.35+3.874(\ln \sqrt{s})+1.146(\ln \sqrt{s})^{2} .
$$

TABLE II. Average multiplicity $\langle N\rangle$ at two extreme pseudorapidity intervals, $|\eta|<0.5$ and $|\eta|<2.4$, at $\sqrt{s}=0.9,2.36$, and $7 \mathrm{TeV}$.

\begin{tabular}{lcrr}
\hline \hline & \multicolumn{2}{c}{ Average charged multiplicity $(\langle N\rangle)$} \\
\cline { 3 - 4 }$|\eta|$ Interval & Energy (TeV) & CMS experiment & Tsallis model \\
\hline 0.5 & 0.9 & $4.355 \pm 0.207$ & $4.583 \pm 0.772$ \\
& 2.36 & $5.262 \pm 0.250$ & $5.489 \pm 0.992$ \\
& 7.00 & $6.808 \pm 0.335$ & $7.409 \pm 1.022$ \\
2.4 & 0.9 & $18.320 \pm 1.273$ & $18.957 \pm 1.174$ \\
& 2.36 & $23.166 \pm 1.716$ & $23.524 \pm 1.382$ \\
& 7.00 & $30.516 \pm 3.660$ & $31.231 \pm 3.042$ \\
\hline \hline
\end{tabular}

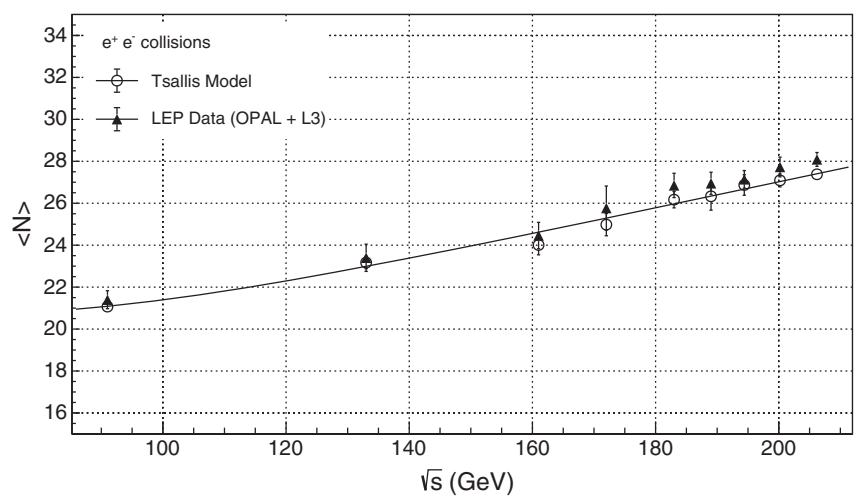

FIG. 1. Dependence of the average multiplicity $\langle N\rangle$ on the center-of-mass energy, $\sqrt{s}$, for $e^{+} e^{-}$collisions and comparison with experimental values. The solid line corresponds to Eq. (16).

Using the Tsallis model, the average multiplicity is predicted for $e^{+} e^{-}$interactions at $\sqrt{s}=500 \mathrm{GeV}$ in the full phase space and for $p p$ interactions at $14 \mathrm{TeV}$ for pseudorapidity range $|\eta|<2.4$.

For $e^{+} e^{-}$interactions at $\sqrt{s}=500 \mathrm{GeV}$, the value of average multiplicity $\langle N\rangle$ is found to be $43.53 \pm 3.79$, whereas for $p p$ collisions, the value of $\langle N\rangle$ at $\sqrt{s}=$ $14 \mathrm{TeV}$ at $|\eta|<2.4$ is found to be $36.18 \pm 3.21$.

\section{B. Moment analysis}

The Tsallis gas model has been used to calculate the moments in order to understand the correlation of the final particles produced during the interaction process. The Tsallis distribution calculated from Eq. (6) is fitted to the experimental data on multiplicity distributions at each of the energies. The multiplicity distribution obtained from the Tsallis model is then used to calculate the moments of the distribution using Eqs. (2) and (3). Figures 3 and 4 show the dependence of normalized and factorial moments on the center-of-mass energy $\sqrt{s}$, calculated by using i) the Tsallis model and also ii) experimental distributions for $e^{+}$

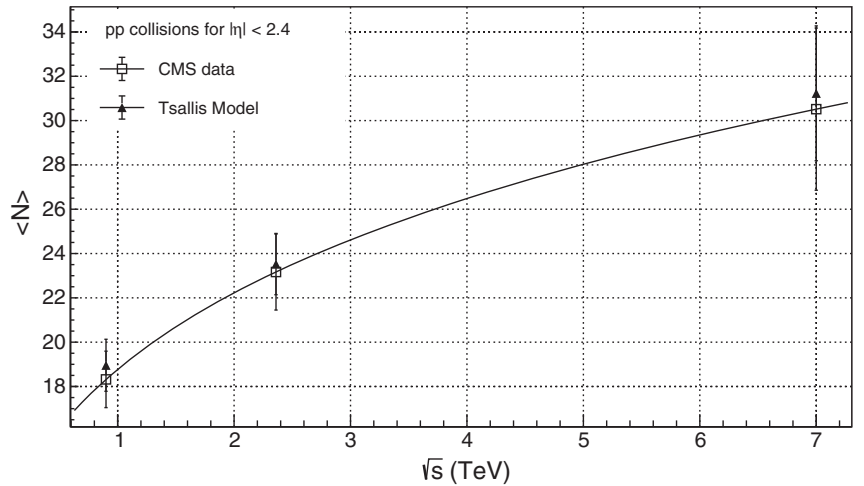

FIG. 2. Dependence of the average multiplicity on the centerof-mass energy. The values from the Tsallis model are compared with the CMS experimental values. The solid line is the fit for the Tsallis model from Eq. (18). 

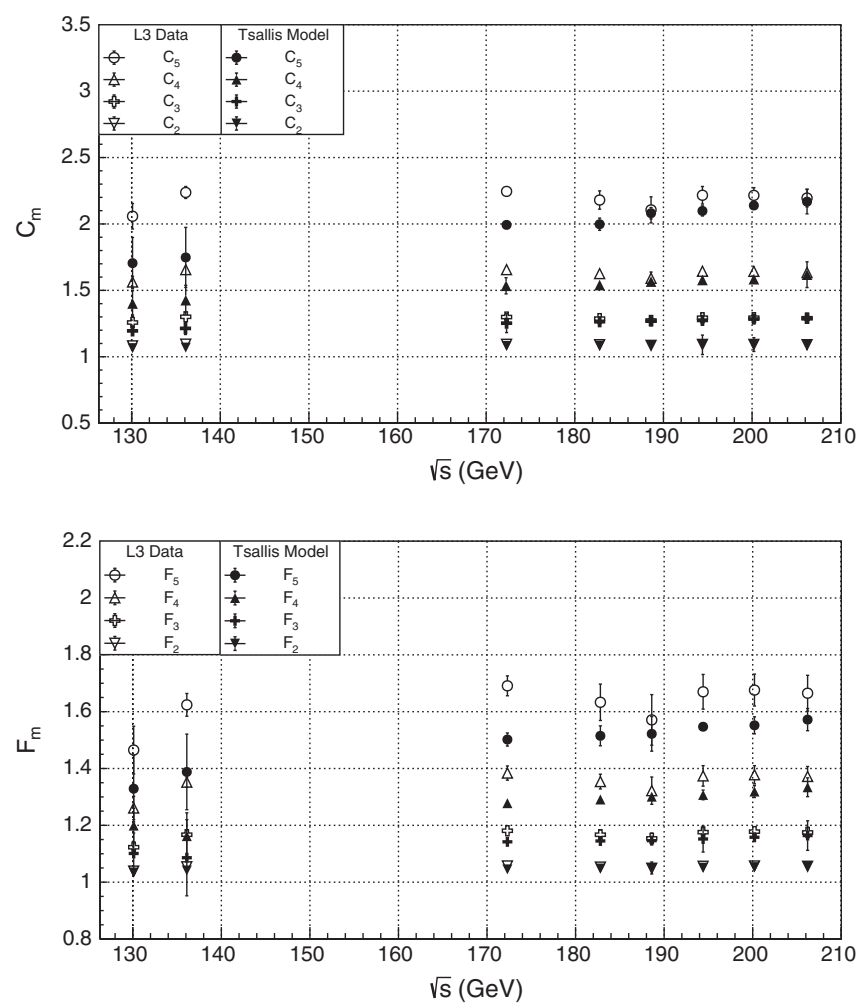

FIG. 3. Dependence of $C_{m}$ and $F_{m}$ moments on the center-ofmass energy, $\sqrt{s}$, and comparison of the moments obtained using the Tsallis model with the L3 experimental values.

$e^{-}$data. The values of these moments are compared with the experimental values and are listed in Tables III and IV. It is observed that the $F_{m}$ and $C_{m}$ moments in each case is nonzero and remains nearly constant with energy.
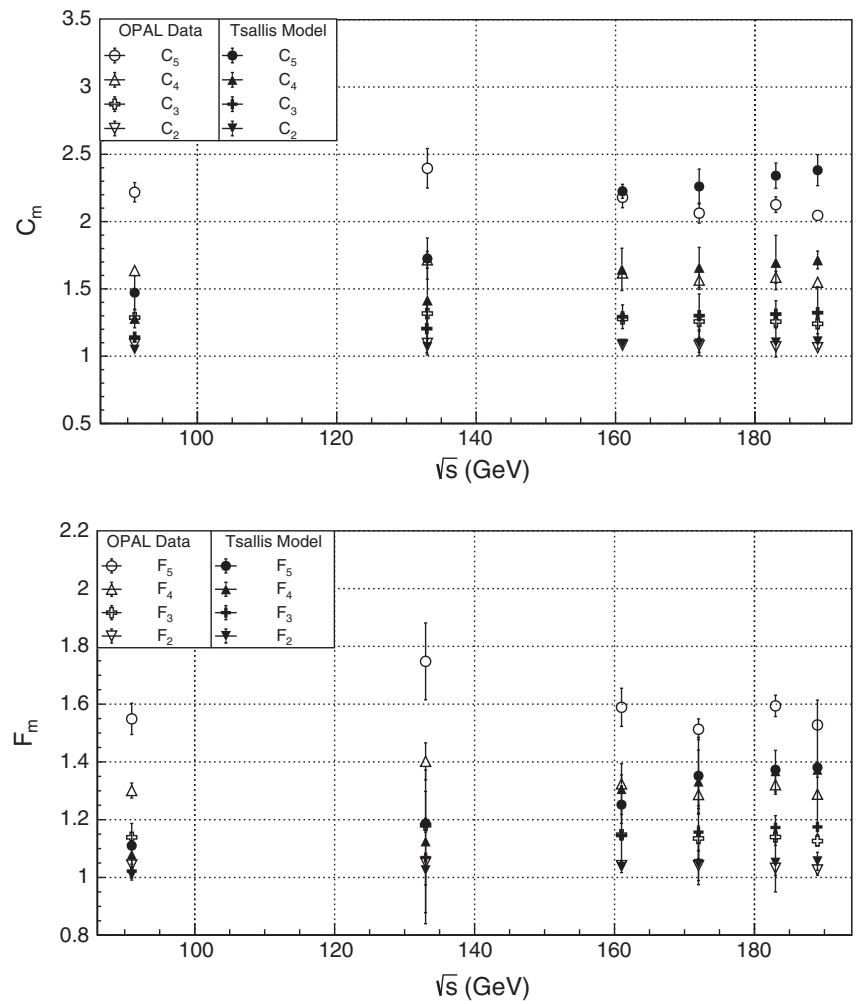

FIG. 4. Dependence of $C_{m}$ and $F_{m}$ moments on the center-ofmass energy, $\sqrt{s}$, and comparison of the moments obtained using the Tsallis model with the OPAL experimental values.

Moments are also calculated for the $p p$ collisions using the CMS data at different pseudorapidity intervals. The dependence of normalized moments, $C_{m}$, and factorial moments, $F_{m}$, on the pseudorapidity, $|\eta|$, at a given energy

TABLE III. $C_{m}$ moments calculated from the Tsallis model for center-of-mass energies, $\sqrt{s}=91$ to $206 \mathrm{GeV}$, for the $e^{+} e^{-}$data.

Energy

$(\mathrm{GeV})$

Experimental reduced moments
Tsallis reduced moments

OPAL experiment

\begin{tabular}{lcccccccc}
\hline & $C_{2}$ & $C_{3}$ & $C_{4}$ & $C_{5}$ & $C_{2}$ & $C_{3}$ & $C_{4}$ & $C_{5}$ \\
\hline 91 & $1.089 \pm 0.003$ & $1.287 \pm 0.012$ & $1.636 \pm 0.029$ & $2.218 \pm 0.072$ & $1.048 \pm 0.011$ & $1.141 \pm 0.032$ & $1.280 \pm 0.069$ & $1.472 \pm 0.127$ \\
133 & $1.095 \pm 0.002$ & $1.317 \pm 0.021$ & $1.716 \pm 0.063$ & $2.396 \pm 0.147$ & $1.068 \pm 0.059$ & $1.204 \pm 0.181$ & $1.416 \pm 0.341$ & $1.725 \pm 0.153$ \\
161 & $1.082 \pm 0.002$ & $1.277 \pm 0.010$ & $1.618 \pm 0.023$ & $2.180 \pm 0.077$ & $1.093 \pm 0.021$ & $1.293 \pm 0.088$ & $1.643 \pm 0.157$ & $2.225 \pm 0.052$ \\
172 & $1.080 \pm 0.052$ & $1.258 \pm 0.061$ & $1.565 \pm 0.069$ & $2.063 \pm 0.074$ & $1.095 \pm 0.091$ & $1.301 \pm 0.161$ & $1.659 \pm 0.149$ & $2.261 \pm 0.129$ \\
183 & $1.070 \pm 0.026$ & $1.257 \pm 0.024$ & $1.586 \pm 0.044$ & $2.126 \pm 0.058$ & $1.102 \pm 0.108$ & $1.313 \pm 0.099$ & $1.696 \pm 0.202$ & $2.341 \pm 0.094$ \\
189 & $1.063 \pm 0.018$ & $1.241 \pm 0.019$ & $1.549 \pm 0.019$ & $2.046 \pm 0.015$ & $1.110 \pm 0.057$ & $1.323 \pm 0.193$ & $1.715 \pm 0.066$ & $2.382 \pm 0.115$ \\
\multicolumn{7}{c}{ L3 experiment } \\
130.1 & $1.082 \pm 0.014$ & $1.258 \pm 0.057$ & $1.563 \pm 0.042$ & $2.058 \pm 0.096$ & $1.065 \pm 0.012$ & $1.195 \pm 0.041$ & $1.401 \pm 0.096$ & $1.705 \pm 0.193$ \\
136.1 & $1.095 \pm 0.002$ & $1.301 \pm 0.007$ & $1.656 \pm 0.019$ & $2.237 \pm 0.045$ & $1.069 \pm 0.015$ & $1.208 \pm 0.046$ & $1.426 \pm 0.112$ & $1.748 \pm 0.226$ \\
172.3 & $1.094 \pm 0.004$ & $1.299 \pm 0.012$ & $1.656 \pm 0.021$ & $2.245 \pm 0.028$ & $1.082 \pm 0.002$ & $1.253 \pm 0.071$ & $1.534 \pm 0.061$ & $1.993 \pm 0.023$ \\
182.8 & $1.091 \pm 0.005$ & $1.287 \pm 0.016$ & $1.626 \pm 0.025$ & $2.180 \pm 0.069$ & $1.084 \pm 0.031$ & $1.264 \pm 0.011$ & $1.540 \pm 0.018$ & $1.998 \pm 0.046$ \\
188.6 & $1.086 \pm 0.007$ & $1.273 \pm 0.020$ & $1.591 \pm 0.047$ & $2.106 \pm 0.098$ & $1.087 \pm 0.011$ & $1.269 \pm 0.013$ & $1.566 \pm 0.027$ & $2.081 \pm 0.043$ \\
194.4 & $1.093 \pm 0.005$ & $1.294 \pm 0.017$ & $1.644 \pm 0.035$ & $2.216 \pm 0.066$ & $1.090 \pm 0.073$ & $1.274 \pm 0.007$ & $1.578 \pm 0.016$ & $2.098 \pm 0.038$ \\
200.2 & $1.093 \pm 0.004$ & $1.294 \pm 0.015$ & $1.643 \pm 0.032$ & $2.215 \pm 0.058$ & $1.092 \pm 0.052$ & $1.284 \pm 0.015$ & $1.584 \pm 0.029$ & $2.139 \pm 0.034$ \\
206.2 & $1.091 \pm 0.006$ & $1.290 \pm 0.016$ & $1.634 \pm 0.035$ & $2.195 \pm 0.067$ & $1.093 \pm 0.009$ & $1.291 \pm 0.036$ & $1.618 \pm 0.097$ & $2.168 \pm 0.092$ \\
\hline \hline
\end{tabular}



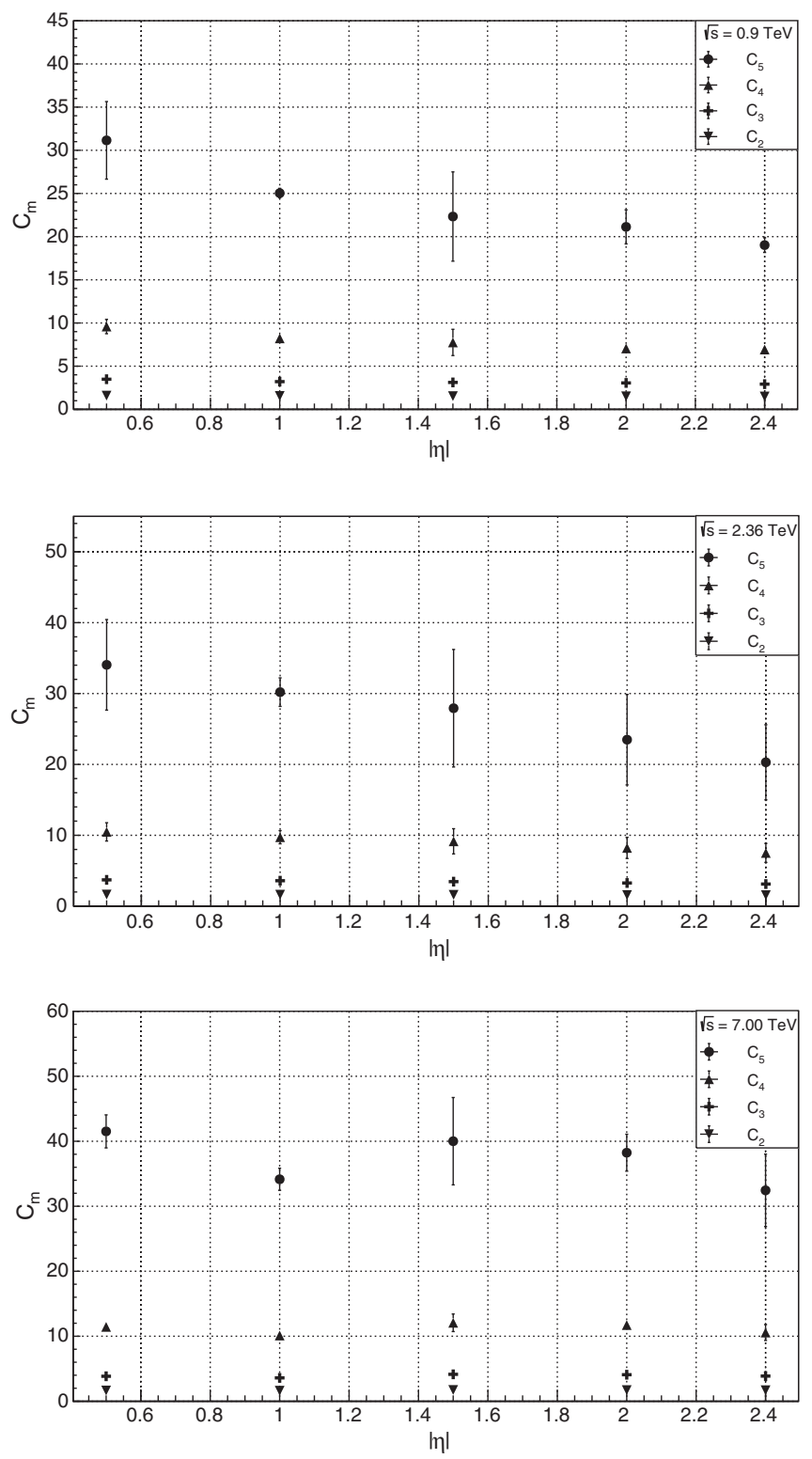

FIG. 5. $C_{m}$ moments obtained from the Tsallis model and its dependence on pseudorapidity intervals $|\eta|$ at $\sqrt{s}=0.9,2.36$, and $7 \mathrm{TeV}$ for the $p p$ data.

and dependence of $C_{m}$ and $F_{m}$ on energy, $\sqrt{s}$ at a given pseudorapidity interval are shown in Figs. 5-8. Figures 5 and 6 show the dependence of the normalized and factorial moments on the pseudorapidity intervals at the given energy [27]. The value of $C_{m}$ decreases with the increase in the pseudorapidity interval at a given energy. This decrease is clearly visible for $C_{5}$ because of its large values. But in the case of factorial moments, the value of $F_{m}$ remains the same within the limit with the increase in the pseudorapidity interval as shown in Fig. 6. Moments obtained from the Tsallis distributions at these pseudorapidity intervals at various energies are compared with the CMS experimental values and are given in Table V. It is found that at each set of pseudorapidity intervals the values
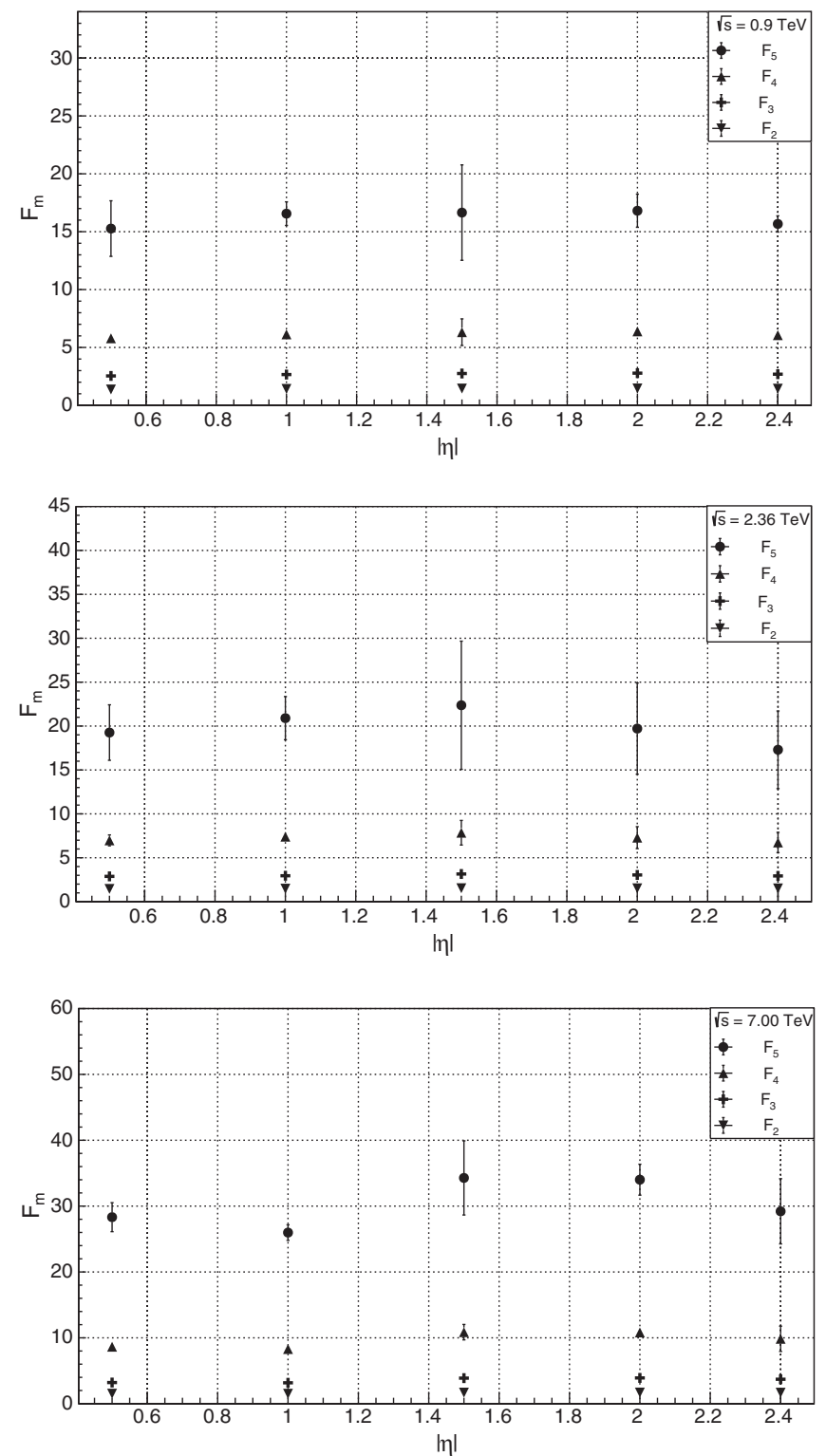

FIG. 6. $F_{m}$ moments obtained from the Tsallis model and its dependence on pseudorapidity intervals $|\eta|$ at $\sqrt{s}=0.9,2.36$, and $7 \mathrm{TeV}$ for the $p p$ data.

of both the moments $C_{m}$ and $F_{m}$ increase with increase in the center-of-mass energy, $\sqrt{s}$, as shown in Figs. 7 and 8. The values of the moments obtained using the Tsallis distribution are found to be in good agreement with the experimental values in both the cases of leptonic and hadronic collisions. In both kinds of interactions, it is found that the factorial moments are larger than unity, which indicates the presence of correlations among particles and deviation from the independent production mechanism.

In the case of leptonic interactions, it is found that the values of normalized moments $C_{m}$ and factorial moments $F_{m}$ are independent of the center-of-mass energy and remain constant with the increase in $\sqrt{s}$ within energy 

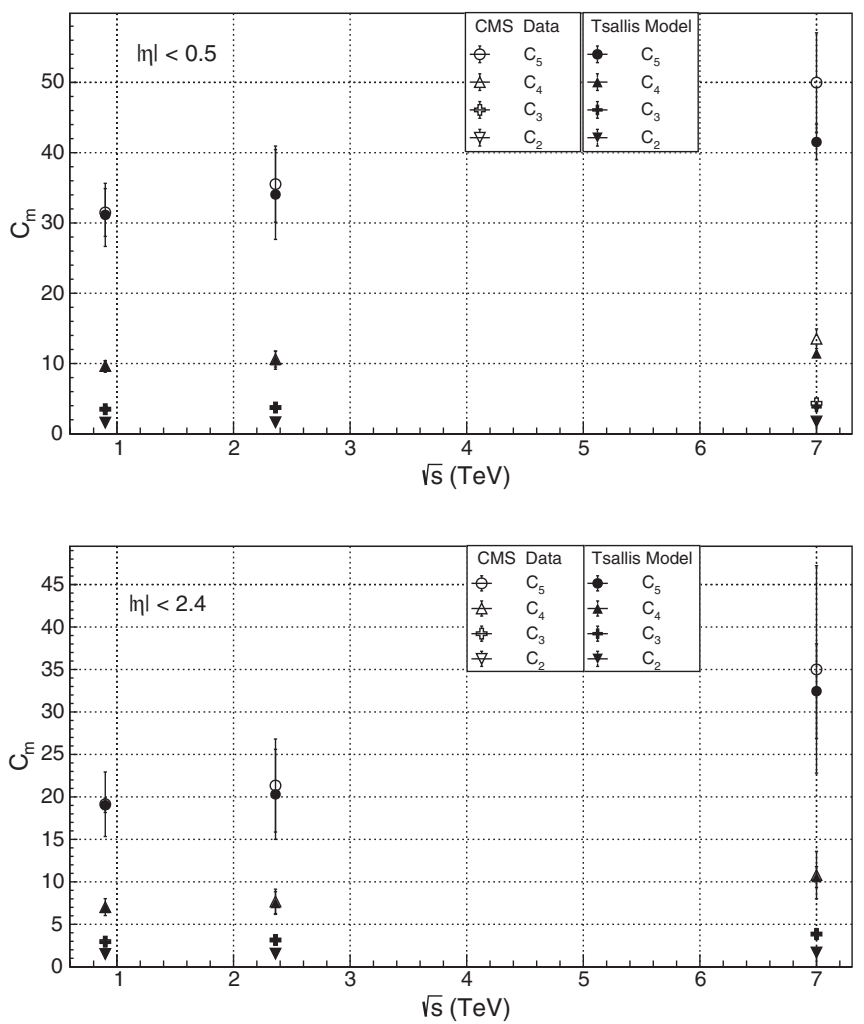

FIG. 7. The variation of $C_{m}$ moments with the center-of-mass energy at pseudorapidity intervals $|\eta|<0.5$ and $|\eta|<2.4$ and comparison of the moments calculated from the Tsallis model with the CMS experimental values [27].
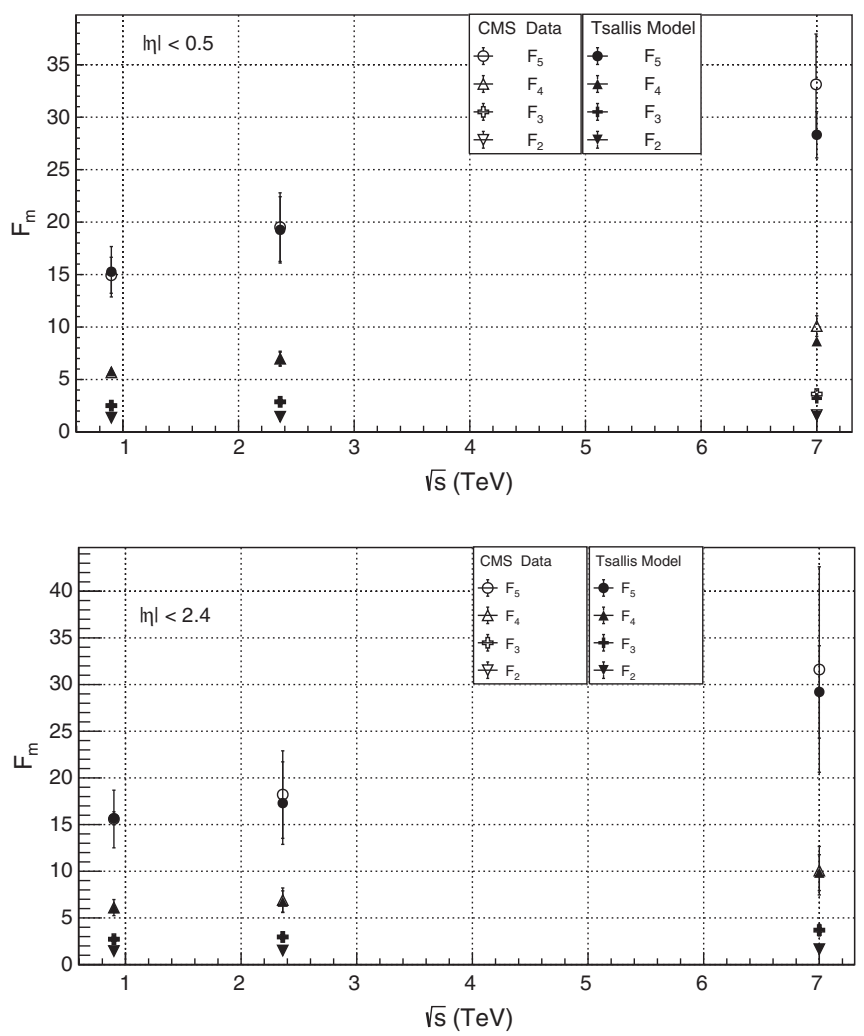

FIG. 8. The variation of $F_{m}$ moments with the center-of-mass energy at pseudorapidity intervals $|\eta|<0.5$ and $|\eta|<2.4$ and comparison of the moments calculated from the Tsallis model with the CMS experimental values [27].

TABLE IV. $\quad F_{m}$ moments calculated from the Tsallis model for center-of-mass energies, $\sqrt{s}=91$ to $206 \mathrm{GeV}$, for the $e^{+} e^{-}$data.

\begin{tabular}{|c|c|c|c|c|c|c|c|c|}
\hline $\begin{array}{l}\text { Energy } \\
(\mathrm{GeV})\end{array}$ & \multicolumn{4}{|c|}{ Experimental factorial moments } & \multicolumn{4}{|c|}{ Tsallis factorial moments } \\
\hline \multicolumn{9}{|c|}{ OPAL experiment } \\
\hline & $F_{2}$ & $F_{3}$ & $F_{4}$ & $F_{5}$ & $F_{2}$ & $F_{3}$ & $F_{4}$ & $F_{5}$ \\
\hline 91 & $1.043 \pm 0.003$ & $1.139 \pm 0.011$ & $1.301 \pm 0.026$ & $1.549 \pm 0.054$ & $1.009 \pm 0.018$ & $1.022 \pm 0.024$ & $1.079 \pm 0.048$ & $1.110 \pm 0.077$ \\
\hline 133 & $1.052 \pm 0.002$ & $1.181 \pm 0.024$ & $1.402 \pm 0.064$ & $1.748 \pm 0.133$ & $1.025 \pm 0.051$ & $1.069 \pm 0.229$ & $1.125 \pm 0.247$ & $1.187 \pm 0.010$ \\
\hline 161 & $1.041 \pm 0.002$ & $1.148 \pm 0.010$ & $1.324 \pm 0.031$ & $1.589 \pm 0.066$ & $1.036 \pm 0.019$ & $1.145 \pm 0.103$ & $1.306 \pm 0.088$ & $1.252 \pm 0.065$ \\
\hline 172 & $1.041 \pm 0.052$ & $1.135 \pm 0.043$ & $1.287 \pm 0.049$ & $1.513 \pm 0.036$ & $1.047 \pm 0.072$ & $1.157 \pm 0.092$ & $1.332 \pm 0.109$ & $1.352 \pm 0.133$ \\
\hline 183 & $1.032 \pm 0.025$ & $1.140 \pm 0.029$ & $1.321 \pm 0.033$ & $1.594 \pm 0.037$ & $1.051 \pm 0.101$ & $1.173 \pm 0.041$ & $1.368 \pm 0.072$ & $1.373 \pm 0.014$ \\
\hline 189 & $1.026 \pm 0.018$ & $1.126 \pm 0.017$ & $1.288 \pm 0.012$ & $1.528 \pm 0.004$ & $1.056 \pm 0.031$ & $1.175 \pm 0.011$ & $1.373 \pm 0.026$ & $1.381 \pm 0.233$ \\
\hline \multicolumn{9}{|c|}{ L3 experiment } \\
\hline 130.1 & $1.039 \pm 0.005$ & $1.123 \pm 0.018$ & $1.261 \pm 0.041$ & $1.465 \pm 0.084$ & $1.034 \pm 0.009$ & $1.101 \pm 0.072$ & $1.199 \pm 0.061$ & $1.329 \pm 0.117$ \\
\hline 136.1 & $1.054 \pm 0.002$ & $1.167 \pm 0.008$ & $1.352 \pm 0.018$ & $1.624 \pm 0.040$ & $1.041 \pm 0.009$ & $1.086 \pm 0.134$ & $1.162 \pm 0.082$ & $1.388 \pm 0.133$ \\
\hline 172.3 & $1.057 \pm 0.005$ & $1.181 \pm 0.014$ & $1.384 \pm 0.025$ & $1.691 \pm 0.035$ & $1.045 \pm 0.003$ & $1.142 \pm 0.006$ & $1.278 \pm 0.014$ & $1.502 \pm 0.023$ \\
\hline 182.8 & $1.053 \pm 0.003$ & $1.167 \pm 0.017$ & $1.354 \pm 0.026$ & $1.633 \pm 0.064$ & $1.048 \pm 0.010$ & $1.145 \pm 0.009$ & $1.291 \pm 0.010$ & $1.515 \pm 0.035$ \\
\hline 188.6 & $1.049 \pm 0.005$ & $1.154 \pm 0.019$ & $1.322 \pm 0.048$ & $1.571 \pm 0.089$ & $1.050 \pm 0.021$ & $1.146 \pm 0.008$ & $1.301 \pm 0.003$ & $1.522 \pm 0.061$ \\
\hline 194.4 & $1.056 \pm 0.006$ & $1.176 \pm 0.018$ & $1.374 \pm 0.036$ & $1.670 \pm 0.061$ & $1.051 \pm 0.005$ & $1.152 \pm 0.046$ & $1.307 \pm 0.017$ & $1.547 \pm 0.009$ \\
\hline 200.2 & $1.057 \pm 0.005$ & $1.178 \pm 0.016$ & $1.378 \pm 0.032$ & $1.676 \pm 0.056$ & $1.053 \pm 0.013$ & $1.158 \pm 0.011$ & $1.319 \pm 0.021$ & $1.552 \pm 0.030$ \\
\hline 206.2 & $1.056 \pm 0.006$ & $1.175 \pm 0.018$ & $1.372 \pm 0.035$ & $1.665 \pm 0.063$ & $1.056 \pm 0.012$ & $1.164 \pm 0.052$ & $1.334 \pm 0.033$ & $1.572 \pm 0.039$ \\
\hline
\end{tabular}


TABLE V. $\quad C_{m}$ and $F_{m}$ moments calculated from the Tsallis model for different pseudorapidity intervals at center-of-mass energy, $\sqrt{s}=0.9,2.36$, and $7 \mathrm{TeV}$, for the $p p$ data.

Pseudorapidity

interval Tsallis reduced moments

Tsallis factorial moments

\begin{tabular}{|c|c|c|c|c|c|c|c|c|}
\hline \multicolumn{9}{|c|}{$\sqrt{s}=0.9 \mathrm{TeV}$} \\
\hline$|\eta|$ & $C_{2}$ & $C_{3}$ & $C_{4}$ & $C_{5}$ & $F_{2}$ & $F_{3}$ & $F_{4}$ & $F_{5}$ \\
\hline 0.5 & $1.59 \pm 0.02$ & $3.49 \pm 0.14$ & $9.59 \pm 0.83$ & $31.15 \pm 4.49$ & $1.37 \pm 0.01$ & $2.54 \pm 0.01$ & $5.79 \pm 0.21$ & $15.27 \pm 2.40$ \\
\hline 1.0 & $1.55 \pm 0.02$ & $3.21 \pm 0.10$ & $8.22 \pm 0.35$ & $25.04 \pm 0.55$ & $1.42 \pm 0.01$ & $2.66 \pm 0.06$ & $6.13 \pm 0.25$ & $16.56 \pm 1.02$ \\
\hline 1.5 & $1.53 \pm 0.06$ & $3.12 \pm 0.35$ & $7.75 \pm 1.52$ & $22.33 \pm 5.17$ & $1.45 \pm 0.04$ & $2.75 \pm 0.24$ & $6.32 \pm 1.15$ & $16.65 \pm 4.12$ \\
\hline 2.0 & $1.51 \pm 0.03$ & $3.06 \pm 0.10$ & $7.05 \pm 0.44$ & $21.13 \pm 1.97$ & $1.46 \pm 0.02$ & $2.78 \pm 0.08$ & $6.39 \pm 0.34$ & $16.81 \pm 1.43$ \\
\hline 2.4 & $1.49 \pm 0.01$ & $2.92 \pm 0.05$ & $6.93 \pm 0.22$ & $19.02 \pm 0.85$ & $1.44 \pm 0.01$ & $2.69 \pm 0.05$ & $6.06 \pm 0.19$ & $15.67 \pm 0.70$ \\
\hline \multicolumn{9}{|c|}{$\sqrt{s}=2.36 \mathrm{TeV}$} \\
\hline 0.5 & $1.64 \pm 0.03$ & $3.71 \pm 0.23$ & $10.48 \pm 1.29$ & $34.05 \pm 6.39$ & $1.45 \pm 0.01$ & $2.88 \pm 0.09$ & $6.98 \pm 0.62$ & $19.26 \pm 3.16$ \\
\hline 1.0 & $1.62 \pm 0.04$ & $3.59 \pm 0.29$ & $9.73 \pm 0.93$ & $30.20 \pm 2.01$ & $1.48 \pm 0.02$ & $2.95 \pm 0.07$ & $7.40 \pm 0.32$ & $20.90 \pm 2.46$ \\
\hline 1.5 & $1.60 \pm 0.06$ & $3.46 \pm 0.36$ & $9.15 \pm 1.78$ & $27.93 \pm 8.29$ & $1.53 \pm 0.04$ & $3.15 \pm 0.29$ & $7.85 \pm 1.40$ & $22.37 \pm 7.30$ \\
\hline 2.0 & $1.57 \pm 0.05$ & $3.27 \pm 0.32$ & $8.22 \pm 1.49$ & $23.49 \pm 6.38$ & $1.52 \pm 0.05$ & $3.04 \pm 0.27$ & $7.29 \pm 1.25$ & $19.71 \pm 5.19$ \\
\hline 2.4 & $1.55 \pm 0.01$ & $3.12 \pm 0.31$ & $7.51 \pm 1.34$ & $20.30 \pm 5.30$ & $1.51 \pm 0.06$ & $2.93 \pm 0.27$ & $6.74 \pm 1.16$ & $17.30 \pm 4.42$ \\
\hline \multicolumn{9}{|c|}{$\sqrt{s}=7 \mathrm{TeV}$} \\
\hline 1.0 & $1.62 \pm 0.02$ & $3.59 \pm 0.07$ & $10.11 \pm 0.34$ & $34.15 \pm 1.70$ & $1.52 \pm 0.01$ & $3.17 \pm 0.05$ & $8.30 \pm 0.24$ & $25.98 \pm 1.18$ \\
\hline 1.5 & $1.73 \pm 0.04$ & $4.14 \pm 0.26$ & $12.07 \pm 1.35$ & $40.01 \pm 6.72$ & $1.68 \pm 0.03$ & $3.88 \pm 0.22$ & $10.86 \pm 1.17$ & $34.28 \pm 5.63$ \\
\hline 2.0 & $1.71 \pm 0.01$ & $4.08 \pm 0.14$ & $11.72 \pm 0.51$ & $38.24 \pm 2.84$ & $1.69 \pm 0.02$ & $3.91 \pm 0.14$ & $10.82 \pm 0.52$ & $34.01 \pm 2.35$ \\
\hline 2.4 & $1.69 \pm 0.04$ & $3.87 \pm 0.25$ & $10.57 \pm 1.22$ & $32.45 \pm 5.57$ & $1.67 \pm 0.05$ & $3.71 \pm 0.23$ & $9.85 \pm 1.92$ & $29.21 \pm 4.95$ \\
\hline
\end{tabular}

range of 91-206 GeV. However, in the case of hadronic interactions, both types of moments increase with the increase in $\sqrt{s}$, whereby the range of $\sqrt{s}$ extends from $900 \mathrm{GeV}$ to $7 \mathrm{TeV}$. These results clearly point toward an understanding of the behavior of produced particles. This also indicates the violation of $\mathrm{KNO}$ scaling at larger energies. But no violation of KNO scaling is observed at lower energies as indicated by the study of leptonic interactions.

\section{UNCERTAINTIES ON MOMENTS}

Errors on the moments for a given normalized probability distribution, $P_{N}$ (i.e., $\sum_{N} P_{N}=1$ ), having an uncertainty $e_{N}$ can be calculated by the method of partial derivatives. This method is consistent with the assumption that errors on the individual bins are assumed to be uncorrelated. We follow this method as described in Ref. [34],

$$
\begin{gathered}
\frac{\partial C_{m}}{\partial P_{N}}=\frac{N^{m}\langle N\rangle-\left\langle N^{m}\right\rangle m N}{\langle N\rangle^{m+1}} \\
\frac{\partial F_{m}}{\partial P_{N}}=\frac{N(N-1) \ldots(N-m+1)\langle N\rangle}{\langle N\rangle^{m+1}} \\
-\frac{\langle N(N-1) \ldots \ldots(N-m+1)\rangle m N}{\langle N\rangle^{m+1}} .
\end{gathered}
$$

The total is then

$$
E_{m}^{2}=\sum_{N}\left(\frac{\partial X_{m}}{\partial P_{N}} e_{N}\right)^{2},
$$

where $X_{m}$ is $C_{m}$ or $F_{m}$.

\section{CONCLUSION}

Detailed analysis of the data on electron-positron annihilation at energies $\sqrt{s}=91$ to $206 \mathrm{GeV}$ and proton-proton collisions at $\sqrt{s}=0.9$ to $7 \mathrm{TeV}$ in various pseudorapidity regions has been done by using the Tsallis distribution. The particle production in such interactions is not uncorrelated. The dynamical fluctuations arising due to random cascading processes in particle production can lead to correlations among the particles. The study of higher-order moments of the distributions serves as a very important tool to understand these correlations. The deviation from independent production can be understood if the factorial moments are larger or smaller than unity. The violation or holding of KNO scaling at higher energies can also be studied and understood correctly by using the normalized moments. The KNO scaling implies the energy independence of these moments, whereas energy dependence of these moments reflects the KNO scaling violation. The normalized and factorial moments have been calculated using the Tsallis model and compared with the experimental values. The obtained values of moments are found to be in good agreement with the experimental values, within experimental uncertainties. The values obtained from the Tsallis gas model confirm the 
violation of $\mathrm{KNO}$ scaling as observed in the experimental values at higher energies, but no such violations are observed at the lower energies. Also, the average multiplicity values calculated from the Tsallis model have been compared with the experimental values and found to be in good agreement with them. Using the Tsallis model, we have predicted the values of average multiplicities for $e^{+} e^{-}$ collisions at $\sqrt{s}=500 \mathrm{GeV}$ and for $p p$ collisions at $\sqrt{s}=$ $14 \mathrm{TeV}$ in the pseudorapidity region $|\eta|<2$.4. In one of the previous studies, the value of average multiplicity at $\sqrt{s}=$ $13 \mathrm{TeV}$ at pseudorapidity region $|\eta|<2.4$ was predicted by using the Weibull model by A. Pandey et al. [24]. The $\langle N\rangle$ value predicted by the Tsallis model at $\sqrt{s}=14 \mathrm{TeV}$ at pseudorapidity interval $|\eta|<2.4$ is found to be consistent with the value predicted by the Weibull model [24]. The study of moments of multiplicity distributions and dependence of average multiplicity on the energy provides interesting features of particle production and helps in the understanding of the mechanism of particle production at higher energies. It will be interesting to study the behavior of particles produced at higher LHC energies $(\sqrt{s}>13 \mathrm{TeV})$ in the future with the Tsallis model.

S. S. is grateful to the Department of Science and Technology, Government of India for the research fellowship grant.
[1] E. De Wolf, J. J. Dumont, and F. Verbeure, Nucl. Phys. B87, 325 (1975).

[2] F. J. Gilman, Studies in the Natural Sciences (Springer, Boston, MA, 1975).

[3] M. L. Mangano, Phys. Usp. 53, 109 (2010).

[4] N. Dobrotin, International Cosmic Ray Conference 10, 456 (1977).

[5] I. M. Dremin, Phys. Usp. 37, 715 (1994).

[6] I. Dremin, Phys. Lett. B 341, 95 (1994).

[7] I. M. Dremin and J. W. Gary, Phys. Rep. 349, 301 (2001).

[8] M. Althoff et al., Z. Phys. C 22, 307 (1984).

[9] K. Goulianos, Gleb Wataghin School on High Energy Phenomenology, Campinas, Brazil, 1994, http://lss.fnal .gov/archive/1994/conf/Conf-94-266-E.pdf.

[10] A. Capella, I. M. Dremin, V. A. Nechitailo, and J. T. T. Van, Z. Phys. C 75, 89 (1997).

[11] M. Praszalowicz, Phys. Lett. B 704, 566 (2011).

[12] C. Tsallis, J. Stat. Phys. 52, 479 (1988).

[13] S. Sharma, M. Kaur, and S.Thakur, Int. J. Mod. Phys. E 27, 1850101 (2018).

[14] S. Sharma and M. Kaur, Phys. Rev. D 98, 034008 (2018).

[15] S. Sharma, M. Kaur, and S. Thakur, Phys. Rev. D 95, 114002 (2017).

[16] S. Sharma, M. Kaur, and Sandeep Kaur, Int. J. Mod. Phys. E 25, 1650041 (2016).
[17] M. Derrick et al., Phys. Rev. D 34, 3304 (1986).

[18] Z. Koba, H. B. Nielsen, and P. Olesen, Nucl. Phys. B40, 317 (1972).

[19] G. J. Alner et al., Phys. Lett. B 160, 199 (1985).

[20] R. E. Ansorge et al., Z. Phys. C 43, 357 (1989).

[21] J. F. Grosse-Oetringhaus and K. Reygers, J. Phys. G 37, 083001 (2010).

[22] N. Suzuki, M. Biyajima, and N. Nakajima, Phys. Rev. D 54, 3653 (1996).

[23] I. M. Dremin, arXiv:hep-ph/0404092.

[24] A. Pandey, P. Sett, and S. Dash, Phys. Rev. D 96, 074006 (2017).

[25] L. Boltzmann, Annalen der Physik 258, 291 (1872).

[26] C. E. Aguiar and T. Kodama, Physica (Amsterdam) 320A, 371 (2003).

[27] CMS Collaboration, J. High Energy Phys. 01 (2011) 79.

[28] P. D. Acton et al., Z. Phys. C 53, 539 (1992).

[29] G. Alexander et al., Z. Phys. C 72, 191 (1996).

[30] K. Ackerstaff et al., Z. Phys. C 75, 193 (1997).

[31] G. Abbiendi et al., Eur. Phys. J. C 16, 185 (2000).

[32] P. Achard et al., Phys. Rep. 399, 71 (2004).

[33] W. Thome et al., Nucl. Phys. B129, 365 (1977).

[34] J. F. Grosse-Oetringhaus and K. Reygers, J. Phys. G 37, 083001 (2010). 